\title{
Utilidad de la Anamnesis en la Detección de Trastornos de la Coagulación
}

\author{
Dra. María Eugenia Hubner G. ${ }^{1}$; Dra. Mireya Bravo L. ${ }^{2}$; Dra. María Morales G. ${ }^{2}$ \\ T.M. Mónica Vera o. ${ }^{2} ;$ T.M. Isene Rojas A, ${ }^{2}$ \\ Abnormal Coagulation: Predictive Value of the Clinical History
}

\begin{abstract}
We studied 104 patients, who underwent preoperatory coagulation tests in the months of June and July 1984. They were all asked for personal and familiar haemorragic antecedents. Ain átmost $94 \%$ positive correlation was found between negative personal history and laboratory results in patients without haemorragic defects. We also studied in retrospective the same paraneters in patiens with Von Willebrand's disease or abnormal platelet's function or number, all under control in our hospital, confirming that in patjents with severe abnomalities of coagulation there always exists some evidence of multisystemic haemorrages. In patients with minor coagulation problems there also appears a history of bleeding. In some mild cases evidence of disease came only from more complex laborstory tests. No statistically significant differences were found in the lvy's bleeding time of patients with previous aspirin ingestion in comparisson with those without it. Careful history taking has high predictive value in the search of coagulation abnormalities.
\end{abstract}

(Key words: Anamnesis. History taking. Bleeding disorders. Coagulation abnormalities).

Si bien es cierto que en la actualidad es posible el diagnóstico preciso de las alteraciones más graves de la coagulación, no es fácil identifi. car las menos severas, que se manifiestan solamente después de traumatismos o intervenciones quirúrgicas. Esto es particularmente import an te en niños que por su corta edad no han estado expuestos a incidentes que pongan en evidencia el trastorno. Por esta razón se solicitan rutina. riamente estudios básicos de coagulaciôn antes de realizar procedimientos de cirugia electiva; así se pretende garantizar un riesgo bajo o nulo de sangramiento durante la operación.

Conocemos la incidencia de trastomos graves de la coagulación en nuestro medio, pero no la de los defectos mínimos. Tampoco se ha establecido la relación entre antecedentes personales y famjliares de sangramiento y los exámenes básicos de coagulación. Consideramos útil hacer un análisis de la correlación en pacientes pediátricos, con el fin de aclarar estas interrogantes.

\section{PACIENTES Y METODOS}

Prospectivamente se compararon los antece-

1. Médico Becario, Depto. Pediatria. Div. Ciencias Médicas Norte. Facultad de Medicina, Universidad de Chile. Hospital Roberto del Rio.

2. Servicio de Hematología. Hospital Roberto del Río. dentes personales y familiares de sangramientos y la historia de ingestión, en los últimos 15 días, de algunas drogas como ácido acetilsalisílico (AAS), fenilbutazoma, indometacina, carbenicilina y fenjotiazínicos, con los resultados de un estudio básico de coagulación, en 104 pacientes enviados para estudios previos a cirugía otorrinolaringológica, en los meses de junio y julio de 1984 .

Los antecedèntes de anamnesis fueron tomados y registrados mediante una encuesta diseñada con anticipación. Los exámenes de coagulación consistieron, en todos los casos en tiempo de sangría (Ivy), tiempo de tromboplastina parcial, tiempo de protrombina y recuento de plaquetas, realizado siempre por las mismas personas y con técnicas estándar $2,3,4$.

En 3 de 4 pacientes cuyo tiempo de sangria estaba prolongado en relación con el antecedente de ingestión de AAS se estudió también la agregación plaquetaria.

Retrospectivamente se compararon los mismos antecedentes de anamnesis y los resultados de los estudios de coagulación en 39 pacientes con Enfermedad de Von Willebrand (EVW) y 14 sujetos con trombopatías confirmadas por los criterios habitualmente aceptados y controlados por la Unidad de Hematología del Hospital Ro. berto del Río.

El valor predictivo de la anamnesis con respec- 
to a los resultados de las pruebas de laboratorio fue analizado mediante la fórmula de Galen ${ }^{5}$.

\section{RESULTADOS}

Seis de los 104 pacientes en que se hicieron exámenes de coagulación como procedimiento preoperatorio, tenían antecedentes de epistaxis de esçasa cuantia, ocasional, que cedía espontá. neamente, alteración que está dentro de lo normal; 2/104 tenían antecedentes de trastornos de la coagulación en algún familiar (Uno tenía 4 tíos maternos con hemofilia $A$ y el otro una tja matema con EVw).

En 37/39 pacientes con EVW se encontrason abundantes antecedentes de hemorragias, siendo lo más frecuente las epistaxis repetida, abundantes de dificil control que necesitan tratamiento médico. Le siguen en frecuencia la formación espontánea de hematomas con traumatismos mínimos, de dificil resolución y gingivorragias o sangramientos profusos después de extracciones dentarias; $6 / 39$ de estos pacientes tenían antecedentes familiares de la enfermedad. Sólo en dos casos no había historia de sangramiento, el diagnóstico derivó de los resultados de los exámenes de laboratorio del estudio pre-operatorio.

En todos los pacientes con trombopatías $(n=14)$ se encontró en la historia antecedentes de sangramientos frecuentes e importantes, includo epistaxis, gingivorragias y hematomas.

Los estudios de coagulación en los 104 pacjentes se resumen en la Tabla 1 y sus resultados están dentro de rangos normales. No se encontraron diferencias estadísticamente significativas en los promedios de tiempo de sangría de Ivy entre los pacientes con y sin antecedentes de haber recibido ácido acetil salicílico. Sólo en 4 pacientes, todos con antecedentes de ingestión de aspirina, el tiempo de sangría de Ivy estaba prolongado, en ellos se indicó no ingeri ninguna droga, $y$ se repitieron las pruebas 21 días después, encontrándose valores normales.

En 3 de estos 4 pacientes se realizó además, estudio de agregación plaquetaria con resultados normales.

Todos los pacientes con trombopatías y EVW tienen un estudio completo de coagulación, según criterios establecidos, que fundamenta los respectivos diagnósticos.

\section{COMENTARIOS}

Estos resultados confirman que la historia dirigida y cuidadosa, tiene alto valor predictivo ${ }^{5}$ y sensibilidad en la detección de ciertos trastor-
Tabb 1

Resultado de pruebas de coagulación en 104 pacientes en preoperatorio de cirugía otorrinolaringológica

\begin{tabular}{|c|c|c|c|}
\hline & & $\begin{array}{l}\operatorname{Sin} A A S \\
64 / 104\end{array}$ & $\begin{array}{l}\text { Con AAS* } \\
40 / 104\end{array}$ \\
\hline \multirow[t]{4}{*}{ Tiempo sangría de Ivy } & $\bar{x}$ & $246,6^{\prime \prime}$ & $249,9^{\prime \prime}$ \\
\hline & $\mathrm{DE}$ & 79 & 118 \\
\hline & $\mathrm{EE}$ & 9,9 & 7,4 \\
\hline & \multicolumn{3}{|c|}{$p=0,99$} \\
\hline T.T.P.K. (seg.) & $\overline{\mathbf{x}}$ & $28,5 "$ & $\left(22^{\prime \prime}-35^{\prime \prime}\right.$ \\
\hline T. Protrombina (seg) & $\hat{\bar{x}}$ & $96,5^{\prime \prime}$ & $(76 "-100 ")$ \\
\hline Rec. Plaquetas $\left(\times 10^{9} 1\right)$ & $\bar{x}$ & 308 & $(179-523)$ \\
\hline
\end{tabular}

AAS: Acido Acetil-salicílico $\bar{X}$ : promedio

DE: Desviación estándars. EE: Error estándar.

nos de la coagulación. La información obtenida mediante su empleo correcto puede ser más relevante ${ }^{1,7}$ que la del examen físico 0 e] resultado de pruebas de coagulación mal elegidas, como el tiempo de sangría Duke y la prueba de coagulación en vidrio 6,7 .

Para una adecuada interpretación de los antecedentes de sangramiento, es importante considerar el tipo de sangramiento, su frecuencia y severidad, si es espontáneo o secundario a traumatismos, si está asociado con alguna enferme. dad sistémica, si tiene relación con la ingestión de ciertas drogas y si existen antecedentes familiares que sugieran transmisión vertical $\mathbf{u}$ horizontal de la alteración ${ }^{4}, 7$. El interrogatorio debe ser bien preparado y formulado de modo que conduzca a una información más completa y mejor 1

En los pacientes con trastornos graves de la coagulación, la historia cuidadosa muestra ten. dencias hemorragiparas multisintomáticas 4,1 siendo comunes epistaxis de difícil control, gingivorragias, hematomas profundos, entre otros. En estos pacientes los exámenes básicos de coagulación están claramente alterados.

En los pacientes con afecciones medianas o leves aparecen también antecedentes de sangramiento en el interrogatorio, salvo excepciones, correspondiendo éstas en su mayor parte a niños que no han tenido oportunidad de tener manifestaciones porque todavía no han cambiado los dientes o no han sufrido traumatismos.

Los pacientes con alteracciones leves de la coagulación pueden tener resultados normales en los exámenes corrientes de laboratorio, apareciendo éstas sólo con estudios más complejos. Aún más, muchas veces sus anormalidades no son 
permanentes sino fluctuantes y los exámenes pueden dar resultados contradictorios ${ }^{6} 9$.

En nuestros 104 pacientes pre-operatorios los exámenes de laboratorio estuvieron dentro de rangos normales y no mostraron diferencias estadísticas significativas en los promedios de $T$. de sangría de lvy cuando se los separó en sujetos con y sin antecedentes de haber recibido ácido acetil-salicílico en los 15 días anteriores a la obtención de las muestras. Sólo 4 pacientes, con antecedentes de ingestión de aspirina tenian timpo de sangría prolongado. Se sabe que ésta produce un defecto en la agregación plaquetaria a través de la acetilación irreversible del sistema ciclo-oxigenasa ciclica y tromboxano $\mathrm{A}_{3}{ }^{1}, 4,7$, 9, 10, 11, y en muy altas dosis, puede inhibir además la síntesis de las prostaglandinas de la pared de los vasos sanguíneos. El efecto de la aspirina es-dependiente de las dosis, pero cantjdades tan pequeñas como $325 \mathrm{mg}$ orales pueden producir alteraciones de la agregaciôn plaquetaria por períodos mayores que una semana ${ }^{11}$. En nuestros pacientes, con antecedentes de ingestión de aspirina y tiempo de sangría de lvy prolon. gado, en el estudio realizado 21 días después de suspender la droga, se encontraron valores normales.

En resumen, la hístoria cuidadosa, dirigida, bien realizada es de alto valor permitiendo predecir, si no hay antecedentes, que los resultados de laboratorio nán normales en alrededor de $94 \%$ de los casos.

Si la historia es positiva con sangramiento leve, el estudio básico de laboratorio puede ser normal y sólo estudios más especializados podrản asegurar que no hay un trastorno de la coagulacjón.

\section{RESUMEN}

Se estudiaron 104 pacientes que acudieron al laboratorio de Hematología del Hospital Roberto del Río para exámenes de coagulación pre-operatorios. Todos fueron sometidos a una encuesta dirigida, previamente confeccionada, buscando antecedentes hemorrágicos personales $y$ familiares, con el fin de conocer la correlación y el valor predictivo de la anamnesis en relación al estudio básico de coagulación. Hubo alta corre. lación positiva entre la historia personal y los resultados de los exámenes de laboratorio en los pacientes sin trastornos de coagulación. Sólo 4/104 pacientes tenían tiempo de sangría prolongado, todos habían ingerido ácido acetil-salicílico recientemente, sus pruebas de agregación plaquetaria fueron nòrmales y el tiempo de sangría se había recuperado 21 días despues.

En 39 pacientes con enfermedad de Von Willebrand confirmada y en 14 sujetos con otros trastornos de la función o el número de plaquetas encontramos evidencia de sangramiento en la historia en 37/39 y 14/14 respectivamente.

Nuestros resultados subrayan la importancia de una buena historia clínica en la detección de trastornos de la coagulación.

\section{REFERENCIAS}

1. Bachmann $F_{\text {.: }}$ Diagnostic approach to mild bleeding disorders. Semin. Hematol 17: 292, (oct.) 1980.

2. Ríos R., Pinochet M., Mandujano M.: Tiempo de sangria de Iyy modificado: valores normales en edad pediátrica. Rev. Chil. Pediatr. 53: 552, 1982.

3. Procedimtentos y técnicas de laboratorio. Vol IV Química Clínica, Parasitología y Hematología. Instituto Salud Pública de Chile. Santiago pp. 47, I983.

4. Kandhasamy Jagathombal; Grünwald H.W., Rosner $F_{\text {.: }}$ Evaluation and management of the bleeding patient. Med. Clin. North Am. 65: 133, 1981.

5. Galen R.S.: The predictive value of laboratory testing Orthop Clin. North Am. 10: 287, 1179.

6. Harber L.A., Slechter S.J.: The bleeding time as a screening test for evaluation of platetet function. $N$. Engl. J. Med. 287; 155, 1972.

7. Malpass T.W., Harber L.A.: Acquired disorders of platelet function. Semin. Hematol. 17: 242, 1980.

8. Ingram G.I.C., Kingsfon P.J., Leslie J, Bowie J.W.: Fold cases of acquited Von Willebrand's syndrome. Br. J. Haematol 21: 189, 1971.

9. Stuart M.J., Merril L., Miller F., Davey R., Wolk $J . A .:$ The post-aspirin bleeding time: a screening test for evaluation hemostatic disorders. Br. J. Haematol 43: 649, 1979.

10. Crapek E.E., Daykin D., Salzman E., Lian E. Hellerstein L.J., Rossodd Ch.B.: Intermediate syndrome of platelet dysfunction. loo- 52: 103, 1978.

11. Sandford J.S., Bennett J.S.: Platelets and their membranes in hemostasis: physiology and pathophysiology. Ann Intern Med. 94: 108, 1980. 